\title{
Aportes de las prácticas educativas implementadas por madres líderes al reconocimiento de sujetos sociales
}

\author{
Sandra Patricia González Zabala ${ }^{1}$ \\ Naví Argentina Rodríguez Rivera²
}

\section{RESUMEN}

El presente artículo, es resultado del trabajo investigativo desarrollado con madres líderes del Programa Más Familias en Acción- Medellín Solidaria, pertenecientes a la comuna 13- San Javier de la ciudad de Medellín. El cual, tuvo por objetivo realizar un acercamiento a los significados construidos por éstas frente a su rol y como lo anterior contribuye a la superación de la pobreza extrema, posibilitando así, la generación de reflexiones que permitieran consolidar y afianzar cada vez más el acompañamiento que se viene realizando con cada una de ellas. Lo anterior, permitió identificar como el liderazgo construido por las mujeres está relacionado con su capacidad para creer, dirigirse, auto-liderarse y ser agentes de su propio desarrollo. Simultáneamente, estos aspectos, les permiten ir confluyendo procesos organizativos y comunitarios en sus territorios, buscando transmitir y transformar desde las necesidades evidenciadas en sus propios hogares, pero también desde el interés por construir maneras de encuentro alrededor de espacios que son comunes y de descubrir potencialidades en dicho camino.

Palabras clave: Desarrollo humano, Liderazgo, comunidad, pobreza, agencia, capacidades.

Recibido: 10 de diciembre de 2014

Aceptado: 10 de marzo de 2015

1 Trabajadora Social Universidad de Antioquia/docente de cátedra de Trabajo Social, Universidad Pontifica Bolivariana. Correo Electrónico: sandra.gonzalez.zabala@gmail.com

2 Master en Investigación Social Cualitativa y Políticas Públicas con experiencia en el campo de los derechos humanos (género, educación, derecho a la tierra, organizaciones sindicales, comunicación en salud sexual y reproductiva, adolescencia y niñez). Facilitadora de ABACOenRed y de procesos de Formación en UPOLI-Managua y organismos no gubernamentales en las Segovias-Nicaragua. Correo Electrónico: naviargentinarodriguezrivera@gmail.com 


\title{
Contributions of educational practices implemented by leader mothers to the recognition of social subjects
}

\begin{abstract}
This article is the result of the research study carried out with leader mothers from the program More Families in Action that inhabit the sector 8 of the city of Medellín; whose objective was focused on understanding the components of educational practices that were implemented in their communities and contributed to their recognition as social subjects. The results of the study show that the leaders discovered themselves as social subjects in relation to their personal development, recognition of the impact of their actions on social transformation, and the desire to know and meet others; as they were developing educational practices with program beneficiaries and their communities.
\end{abstract}

Keywords: Subjects, educational practices, experiences, popular education, social transformation. 


\section{INTRODUCCIÓN}

Los Programas de Transferencias Monetarias Condicionadas han sido implementados en América Latina como una estrategia para superar la pobreza e interrumpir su reproducción intergeneracional, todo a través de la entrega de un incentivo monetario periódico dirigido a los hogares más vulnerables de los países de la región; siempre que éstos cumplan con unas condiciones preestablecidas por el propio programa en lo que respecta a la inversión en el capital humano de las mujeres, los niños y las niñas.

En Colombia, se inicia con la implementación de este tipo de programas aproximadamente en el 2002 con Familias en Acción, hoy denominado Más Familias en Acción; con el objetivo de promover la salud y la educación de los menores de edad para que los grupos familiares dispusieran de un complemento al ingreso que permitiera el cumplimiento de dicho objetivo.

Este programa desarrolla un componente de promoción que es dinamizado por las madres líderes, quienes a su vez son beneficiarias del programa y realizan su trabajo de manera voluntaria durante dos años y una vez finalizado su periodo, son reemplazadas por otro grupo de mujeres. Sin embargo, en el caso de la ciudad de Medellín, las mujeres pueden permanecer en el proceso, si así lo desean, acompañando a las nuevas líderes con sus aprendizajes y experiencias vividas.

En la ciudad de Medellín, el componente de promoción toma el nombre de Acompañamiento Grupal y se desarrolla en todo el territorio municipal, por lo que hay madres líderes y madres líderes acompañantes en todas las comunas y los cinco corregimientos de la ciudad. Dicho acompañamiento se encuentra bajo la responsabilidad de un equipo de profesionales de las ciencias sociales, quienes desarrollan junto con las participantes todas las fases contempladas en el proceso.
El interés por trabajar las prácticas educativas desarrolladas por estas líderes surge de la evidencia clara del proceso de evolución que tuvieron en su desarrollo personal como mujeres a lo largo de su participación en el acompañamiento grupal del programa, lo cual les permitió protagonizar procesos de transformación al interior de sus familias y de sus comunidades. Por lo tanto, era necesario indagar por aquello que había detrás de dichas prácticas educativas, que llevaba a las líderes a reconocerse como sujetos en la praxis social.

\section{MATERIALES Y MÉTODOS}

Indagar por la configuración de sujetos sociales, en el marco de programas sociales a partir de la implementación de prácticas educativas de las líderes, implica ubicar el estudio investigativo desde una postura socio-crítica que asume como opción ética y política la construcción de conocimiento para aportar a los procesos de transformación social.

Por consiguiente, el interés está centrado en contribuir y potenciar su rol como agentes de cambio, y en la búsqueda de la emancipación y la liberación de los sujetos que hacen parte de la realidad "objeto de estudio"; en primer lugar, reconociéndolas como seres humanos que hacen parte de un grupo social que ha sido excluido y marginado de los bienes y servicios que ofrece la ciudad de Medellín; en segundo lugar, visibilizando los contextos situacionales en los que se construye su vida cotidiana; en tercer lugar, develando el enfoque que tiene el gobierno local y nacional en la implementación de las políticas públicas a través del Programa 'Más Familias en Acción' y, finalmente, evidenciando la construcción del ejercicio del liderazgo que han venido haciendo en sus comunidades como una alternativa para el mejoramiento de su calidad de vida desde la participación en el programa encaminada al cambio y la transformación de sus contextos. 
En coherencia con los planteamientos anteriores, este estudio asume como enfoque investigativo la investigación cualitativa, debido a que encuentra su énfasis en la comprensión de las realidades que viven las líderes, la forma cómo se relacionan, sus prácticas y las expresiones en sus contextos naturales, como el familiar y el comunitario.

Los planteamientos anteriores llevan a decidirse por una investigación de corte comunitario que se interroga por la acción, por el trabajo comunitario, por su cualificación desde la perspectiva de quienes participan y especialmente por las prácticas que implementan las líderes en sus comunidades, como agentes de cambios. De acuerdo a Ghiso (2006) la opción por una investigación comunitaria obedece a que "han estado ligadas a los procesos de acompañamiento o intervención, han introducido cierta racionalidad en las prácticas, modificando las formas de comprensión y expresión de las mismas; han permitido reinformar la práctica y cualificar contenidos o componentes, y han contribuido a sistematizar los procesos de intervención, de modo que el trabajo comunitario se convierte en una acción críticamente informada y coherente con los intereses y fines que persigue".

De manera complementaria, esta investigación se orienta desde una perspectiva de Investigación Acción Participación (IAP) en lo que refiere a la promoción de la participación como una posibilidad para la construcción colectiva del conocimiento; la intencionalidad transformadora que tiene como marco orientador del proceso investigativo y la producción de conocimientos que contribuyan a aportar a la potenciación de las practicas comunitarias y resolución de los problemas que se presentan.

Esta investigación se desarrolló en tres fases; exploratoria, focalización y profundización, la primera, corresponde a la construcción del estado del arte, la búsqueda de información sobre los temas relacionados al objeto de estudio, las inmersiones de campo iniciales para observar las prácticas de liderazgoy del entorno de la comuna 8 y la revisión de documentos escritos del proceso de acompañamiento grupal como actas, memorias, informes y guías de trabajo.

En la fase de focalización se desarrolló; un taller investigativo, 3 entrevistas semiestructuradas, y la observación participante en los escenarios naturales de las líderes para profundizar en la información existente. Así mismo, se realizó el proceso de organización, tematización, categorización y análisis de la información generada en el estudio que posibilitara el ir y venir, así como la contratación de toda la información.

Finalmente en la fase de profundización, se elaboró un primer informe para avanzar en la socialización y validación con las participantes del proceso, permitiendo afinar los hallazgos en relación con las categorías de análisis establecidas.

\section{RESULTADOS Y DISCUSIÓN}

En coherencia con el objeto de estudioy la metodología utilizada, se tomó la opción de elegir planteamientos teóricos coherentes con el enfoque crítico social, toda vez que la reflexión que se genere, debe contribuir a la cualificación de la práctica social y al empoderamiento de las mujeres líderes del programa.

En este sentido, la fuente principal de la cual bebe esta investigación es la educación popular ya que pone en el centro de las prácticas sociales al sujeto; otorgándole poder y protagonismo, en tanto son sus intereses, sus saberes, sus necesidades y sus aspiraciones los que lo movilizan a emprender procesos de transformación de sus condiciones de vida en compañía de los otros. De igual manera, porque se reconoce el carácter político de la educación, asumiendo una opción explicita por la búsqueda de una sociedad más justa y democrática. 
Las prácticas educativas visibilizadas por las madres líderes como posibilitadoras del ejercicio de su liderazgo se describen a partir de la conexión que tienen con sus experiencias vitales desde tres ámbitos: el programa, la familia y el vecindario, en cada uno de ellos es posible evidenciar los actores significativos, la finalidad y la metodología utilizada.

Los tres ámbitos se anudan desde cuatro componentes: la posibilidad de generar espacios de encuentro para compartir, acceder a información para tomar decisiones y participar; el trabajo cooperativo entre los sujetos que participan del proceso y los principios éticos que orientan el ejercicio del liderazgo.

Las prácticas desarrolladas en el ámbito del programa, giran en torno al cumplimiento de los objetivos de Más Familias en Acción, específicamente lo relacionado con el bienestar comunitario, cuyos objetivos están centrados en la promoción de la salud, la nutrición y la educación de los menores beneficiarios del programa.

Por esta razón, desde este ámbito se trabaja directamente con las familias beneficiarias para que asuman la salud, la nutrición y la educación de sus hijos como base fundamental para el mejoramiento de sus condiciones de vida; toda vez que se considera que con estas dimensiones satisfechas a los menores se les facilita la construcción de una idea de futuro distinta. Las prácticas realizadas fueron los encuentros de bienestar, las reuniones informativas y las asesorías.

El ámbito de la familia, constituye su espacio más íntimo de relaciones, es allí donde podían ver lo que eran como mujeres desde el lugar de cuidadoras y protectoras de los hijos. De igual manera, el lugar donde era más visible evidenciar los cambios que iban surgiendo de su proceso de participación en el acompañamiento grupal. Por esta razón, se desarrollan al interior de sus grupos familiares.
Dichas reflexiones surgían del proceso personal que estaban haciendo en el marco de su participación en el programa; no sólo fueron los temas trabajados en el proceso formativo los que les pusieron preguntas a su propia vida, sino también el contacto directo con la realidad de otras mujeres que vivían en su entorno más cercano y que se vinculaban a las prácticas educativas que ellas realizaban.

De esta manera, el proceso de transformación fue tocando el ámbito más íntimo de las líderes: su propio ser, expresado en su auto reconocimiento como mujeres autónomas y capaces de alcanzar sus sueños y en las maneras de relacionarse con sus hijas e hijos, así como en la manera de establecer sus relaciones sentimentales. Se dan cambios cuando las mujeres empiezan a pensar en ellas mismas, cuando se dan cuenta que pueden tener control sobre la vida del hogar y reorganizar las relaciones con sus integrantes, haciendo la vida más liviana. Las practicas implemetadas fueron; las micro sesiones educativas y la generación de espacios de conversación

El ámbito vecinal, se refiere al sector del barrio que la líder reconoce como el lugar donde se desarrolla su vida cotidiana. Es un lugar donde hay una lógica propia de organización social determinada por las características socioculturales de los habitantes, por las relaciones que se construyen entre los vecinos y por la realidad que se comparte; el vecindario para las líderes, se constituye en el referente de comunidad en tanto han construido lazos y sentimientos de pertenencia con el lugar donde viven y con sus habitantes.

En este sentido, a medida que su proceso de empoderamiento se fue haciendo más evidente, las líderes empezaron a interesarse por abordar los problemas que vivían las familias desde una perspectiva comunitaria, la cual requería de la participación de todos. Por esta razón, las prácticas educativas empiezan a desarrollarse con todos sus vecinos, independiente si eran beneficiarios del programa o no. 
Fueron tres las líneas temáticas que concentraron el interés de las líderes: las violencias contra las mujeres, la vida de los jóvenes y la violencia intrafamiliar, siendo para ellas los mayores problemas sociales que tenían que enfrentar las familias cotidianamente. Es por ello, que decidieron autónomamente proponer la apertura de espacios de encuentro para reflexionar sobre lo que estaba sucediendo en sus sectores como un aporte para la transformación de la vida de los vecinos como los talleres con familias y los juegos tradicionales para los niños y los jóvenes.

Las prácticas educativas desarrolladas por las líderes desde los diferentes ámbitos, mostraron que la vida se construye explorando, arriesgándose, aventurándose, tejiéndola día a día y también, cuando era necesario, destejiéndola para reconocer el lugar donde estaban y lo que deseaban ser.

En este sentido, se esbozan unos componentes que ofrecen unas pistas para comprender los elementos que confluyeron en el proceso que facilitaron que las líderes fueran descubriéndose como sujetos sociales, así:

El descubrimiento de su ser de mujer, fue uno de los principales componentes que emergió del análisis de las prácticas, en la medida en que las mujeres salieron de sus casas a desarrollar innumerables actividades en su rol comunitario y público y lograron incidir en la vida de su comunidad, comprendieron que había una vida que trascendía el ámbito doméstico.

En este sentido, la transformación real y duradera de la vida de las mujeres y la de su familia solo fueron posible gracias a que el proceso se desarrolló desde sus experiencias vitales, sus preguntas e inquietudes más sentidas. Se trataba de generar las condiciones para su crecimiento personal ofreciendo herramientas para aportar a la construcción de su identidad como mujeres.
Un crecimiento personal, expresado en la movilización de procesos internos que permitieron a las líderes aprender o mejorar artes de cuidado de su vida, reconocer sus derechos, desarrollar la atención sobre los aconteceres diarios, mirarse como mujeres interesantes y significativas, ocuparse de sí mismas y de las personas que amaban con atención permanente y amorosa.

Un segundo componente fue el descubrimiento del conocimiento; de acuerdo con los planteamientos de Vigil (1989), "la vida está condiciona por los conocimientos que los sujetos tienen de la realidad, de los hechos y los acontecimientos que viven". Es por ello, que a través de los conocimientos que un sujeto apropia en primer lugar, es posible delimitar el mundo en que vive, actúa y se mueve; en segundo lugar, identificar los recursos materiales y simbólicos con los que afronta su vida cotidiana $\mathrm{y}$, en tercer lugar, evidenciar la manera como produce y recrea lo que entiende de la realidad.

Por consiguiente, la apropiación del conocimiento tiene una dimensión de poder que se expresa en las maneras como se presentan las interacciones entre los sujetos y en las que suceden entre éstos y su mundo, lo cual no puede concebirse de manera aislada del contexto sociocultural donde ocurre.

Es por ello, que la apropiación del conocimiento para las madres líderes tuvo un carácter situado en el presente, en las necesidades del aquí y del ahora sin perder la dimensión histórica. Desde esta perspectiva, fue posible para ellas desnaturalizar los problemas sociales que padecían al interior de sus grupos familiares y los se presentaban alrededor de la familia en sus comunidades, así como ampliar su comprensión frente a la intencionalidad del programa con la entrega de la transferencia condicionada.

Un tercer componente, asociado al descubrimiento de la incidencia de sus acciones; en las prácticas se 
evidencia claramente la construcción de alternativas sociales y culturales que logran subvertir el orden establecido en las familias y en la comunidad, superando el discurso institucional del programa y avanzando en la transformación de relaciones de poder prevalecientes. Todo ello, se presentó, una vez que las líderes fueron capaces de reconocer y nombrar la realidad que vivían, de movilizar a sus vecinos para participar en el desarrollo de alternativas sociales de transformación y de conquistar espacios de poder que trascendían lo familiar a lo comunitario.

Es preciso anotar, que las líderes pudieron evidenciar la incidencia de sus acciones en la transformación de los espacios microsociales más cercanos a ellas como la familia y el vecindario. Todo ello, reafirma la premisa de Rauber, I (2006) de que el sujeto se revela en el curso de un proceso social que implica el desarrollo de prácticas trasformadoras, la identificación de formas de organización y movilización de la sociedad y la conciencia crítica de la realidad.

Un cuarto componente que refiere al descubrimiento del encuentro con el otro; en contraposición a lo que el modelo neoliberal proclama en relación a la individualización de la sociedad, las líderes descubrieron la potencia del encuentro entre los seres humanos para compartir lo que acontecía en la vida cotidiana.

El encuentro entre sus familias y sus vecinos era la posibilidad de reconocerse como seres humanos de historia, de conocimientos, de saberes y de emociones, capaces de realizar procesos de objetivación con los otros, de producir y convenir un imaginario común, de crear un mundo simbólico en la interacción con el ambiente del cual hacían parte y por lo tanto de construir su propia realidad.

\section{CONCLUSIONES Y RECOMENDACIONES}

Una de las primeras reflexiones que deja esta investigación, está centrada en el reencuentro de las líderes con su ser de mujer a través de los ojos de otras mujeres y de hombres que hacían parte de sus grupos familiares y de suvecindario. El desarrollo de prácticas educativas, implicaba la interacción con el mundo que estaba por fuera del espacio doméstico, el cual las llevaba a resignificar su propia imagen, a resignificar los problemas sociales que se presentaban alrededor de las familias y, a resignificar sus propias relaciones.

Por consiguiente, las líderes vivenciaron un proceso orientado hacia su desarrollo personal, basado en la movilización de procesos internos que facilitaron a las mujeres el aprendizaje del cuidado de sus vidas, la observación consciente de la vida cotidiana, la confianza para caminar por la vida como personas valiosas y con conocimientos; el deseo de ocuparse de sí mismas y de las personas que amaban y la ratificación en su decisión de contribuir a la transformación de su vecindario.

Para profundizar en el proceso con las líderes y facilitar otras herramientas que contribuyan al desarrollo personal de las mujeres, es importante incorporar al acompañamiento grupal, unos lineamientos centrados en el enfoque de género, en razón de avanzar en su empoderamiento $\mathrm{y}$ en la transformación de sus entornos familiares y comunitarios.

Una segunda reflexión, se basa en el alcance de los aportes que hacen las mujeres a la transformación social de sus territorios; resulta interesante evidenciar como las prácticas educativas tienen una influencia en el ámbito microsocial de la vida de las líderes, en tanto es su familia y su vecindario más cercano, el que constituye su espacio de acción y de incidencia.

Es por ello, que es posible concluir que el proceso transformación de los sectores donde habitan las 
líderes, se va tejiendo a partir de acciones concretas en los espacios familiares y en los espacios vecinales para convertirse en una gran red de transformaciones que modifican las relaciones de poder y por tanto tienen un carácter político que se expande por todo el territorio.

La tercera reflexión, está relacionada con el carácter situado de la experiencia de las líderes, es decir, es posible visibilizar unos componentes centrales relacionados con el reconocimiento de las líderes de la comuna 8 como sujetos sociales, en tanto se comprende sus vivencias y sus experiencia en el marco de la dinámica social y cultural del territorio en el que viven.

El acercamiento a las experiencias educativas desde la mirada de las líderes de la comuna 8 , se constituye en el inicio de una reflexión que puede profundizarse con la mirada de otros actores que confluyen en dicha experiencia; los integrantes del equipo de acompañamiento grupal, las directivas del programa, los actores institucionales y líderes de organizaciones presentes en el territorio con los que tienen relación. Todos ellos, tienen algo que decir y expresar sobre el proceso adelantado por la líderes, por lo cual, no cabe duda que pueden encontrarse mayores elementos para comprender su proceso de reconocimiento como sujetos sociales.

De igual manera, sería muy interesante para el programa desarrollar una investigación con las madres líderes que habitan en todas las comunas y corregimientos de Medellín; allí sería posible identificar las múltiples y variadas prácticas educativas que se desarrollan en el territorio y que aportan a la transformación de esta ciudad.

\section{REFERENCIAS}

Bonilla, E y Rodríguez, P. Más allá de los métodos: La investigación en ciencias sociales, Ed Norma, Colombia, 1997, p. 118.
Dávalos, P. Neoliberalismo político y Estado social de derecho, p. 21-22. Recuperado el 11 de Febrero de 2015 en: http://www.puce.edu.ec/documentos/ Neoli beralismoyEstadosocialdederecho.pdf.

Economía del cuidado: Las mujeres y la redistribución del trabajo doméstico. Cuadernos Sobre Economía Desde Las Mujeres $\mathrm{N}^{\circ} 1$, Corporación Vamos Mujer, Medellín - Colombia, Diciembre de 2011.

El Faro, Comuna 8: Diagnóstico y propuestas comunitarias para el mejoramiento integral del barrio. Medellín- Colombia, 2013. Recuperado el 28 de Enero de 2013 en $\quad$ file:///C:/Users/IlilDean/Downloads/Propu estaMejoramientoIntegraldelBarrioE1Faro Comuna $8 \% 20(1)$.pdf

El Trabajo Doméstico a partir del sentir de Mujeres rurales y urbanas. Cuadernos sobre Economía desde las Mujeres $\mathrm{N}^{\mathrm{o}}$ 3, Corporación Vamos Mujer, Medellín- Colombia, mayo 2014.

Encuesta de Calidad de Vida. Medellín, Colombia, 2013. Recuperado el 29 de Enero d e

2015 en http://www.medellin.gov.co/irj/portal/ ciud adanos?NavigationTarget $=$ navurl://4261ad $\underline{61 \mathrm{f} 6 \mathrm{~d} 657977 \mathrm{c} 33 \mathrm{e} 865 \mathrm{fd} 230 \mathrm{~b} 1 \mathrm{e}}$

Estrategias de Educación Popular, Escuela de Formación para la Organización Comunitaria, Ministerio del Poder Popular para Ciencia, Tecnología e Industrias Intermedias, CaracasVenezuela, 2010, p. 11.

Freire, P. Pedagogía del Oprimido. Siglo XXI, Tierra Nueva, Montevideo, 1970, p. 99.

Freire, P. Pedagogía de la Esperanza, un reencuentro con la pedagogía del oprimido, Siglo Veintiuno: Segunda edición, Buenos Aires - Argentina 2011.

García, B y otros. Técnicas interactivas para la investigación social cualitativa, Fundación Universitaria Luis Amigo, Ed Fondo, MedellínColombia, 2002.

Ghiso, A. Rescatar, descubrir, recrear, metodologías participativas en investigación social comunitaria. En: Revista Fundación Universitaria Luis Amigó Vol. 9, $\mathrm{N}^{\mathrm{o}}$ 13, Medellín - Colombia, 2006. 
Ghiso, A. Formar en investigación desde la perspectiva de la educación popular. En: Entretejidos de la educación popular en Colombia, Ediciones desde abajo, CEAAL, Bogotá - Colombia, Febrero de 2013, p. 106.

Kawulich, B. La observación participante como método de recolección de datos. Forum: Qualitative Social Research. Mayo. Volumen 6, No 2, Artículo 43, 2005.

Las mujeres de la comuna 8 emprenden desde lo social. Medellín-Colombia, febrero 06 de 2013. Recuperado el 20 de Enero de 2014 en: http:// www.eafit.edu.co/agencia-noticias/historiconoticias/2013/noticias-febrero/Paginas/proyeccionemprendimiento-social-comuna-8.aspx\#. VMhfnmjLfRM

Mejía, M. La Educación Popular Hoy: Reconstruyendo su identidad desde sus acumulados y en diálogo con la teoría crítica, La Piragua Revista Latinoamericana y Caribeña de Educación y Política. No. 30, CEAAL, 2009, p.46.

Mondragón, G y Ghiso, A. Pedagogía Social. Universidad del Valle: Escuela de Trabajo Social y Desarrollo Humano, Segunda Edición, Santiago de Cali - Colombia, 2010, p.56-57

Pérez, G. Investigación cualitativa retos $e$ interrogantes, Ed La Muralla, Madrid - España, 1998, p.126.

PRIMED (Programa lntegral de Mejoramiento de Barrios Subnormales en Medellín). Una experiencia exitosa en la intervención urbana. Medellín- Colombia, 1996. Recuperado el 01 de Febrero de 2015 en file://C:/Users/IlilDean/ Downloads/Docu mento\%20primed\%20(2).pdf
Protocolo de Acompañamiento Grupal, [versión digital], Programa Medellín Solidaria, MedellínColombia, 2011.

Rauber, I. Movimientos sociales representación politica, Ediciones desde abajo, Bogotá Colombia, 2003, p.63.

Rauber, I. Sujetos politicos, rumbos estratégicos y tareas actuales de los movimientos sociales y políticos en América Latina, Ediciones desde abajo, Bogotá - Colombia, 2006.

Torres, R. Consensos fundamentales sobre las características de la educación popular. En: discursos y prácticas de la educación popular, Quito, 1989.

Torres, A. Sujetos y subjetividad en la educación. En: Pedagogía y Saberes, Universidad Pedagógica Nacional, Facultad de Educación. No. 15, BogotáColombia, 2000, p.7.

Torres, A. La construcción del objeto y los referentes teóricos en la investigación social. La práctica investigativa en ciencias sociales, Bogotá, UPN (Universidad Pedagógica Nacional), 2004, p. 21 -22 .

Torres, A. Educación popular trayectoria y actualidad. Editorial El Buho, Bogotá - Colombia, 2008.

Torres, A. La reactivación de la Educación Popular en el despertar del nuevo milenio, La Piragua: Revista Latinoamericana y Caribeña de Educación y Política. No. 38, CEAAL, Octubre de 2013, p. 37.

Vigil, J. Educación Popular y Protagonismo Histórico: Una Opción para América Latina, Ed. HVMANITAS, Buenos Aires - Argentina, 1989, p. 101. 